\title{
Editorial: Biomaterials for Cell-Based Therapy
}

\author{
Liyuan Zhang ${ }^{1 *}$ and Huanan Wang ${ }^{2 *}$ \\ ${ }^{1}$ School of Engineering and Applied Sciences, Harvard University, Cambridge, MA, United States, ${ }^{2}$ School of Life Science and \\ Biotechnology, Dalian University of Technology, Dalian, China
}

Keywords: cell encapsulation, biomaterials, MSC, stem cell therapy, IPS

Editorial on the Research Topic

Biomaterials for Cell-Based Therapy

Cells are becoming one of the most important and promising forms of therapy. The use of cells as therapeutics has stimulated numerous new therapeutic companies that have reached valuations of billions of dollars. Stem cells, such as embryonic stem cells, iPSCs (pluripotent stem cells), and adult stem cells, are being used to regenerate human tissue for autologous and allogeneic therapy and for autoimmune disease. It offers the perfect solution when there is a need for tissue and organ shortage or organ transplantation, differentiating into specific cell types that are required for tissue repair. Immune cells can be collected directly from patients, engineered for new functionality, and injected into patients as a key component of immunotherapy. For instance, the use of hematopoietic stem cell transplantation can broadly be broken down into autologous cell transplants, represented by $\sim 2,000$ patients per year and $\sim 8,000$ for allogeneic transplants in the US. Cells directly injected into the body are not very effective in performing their tasks and are instead rapidly degraded, making the process very inefficient. Moreover, this often induces graft versus host disease (GVHD), a highly morbid complication that kills allogeneic transplant patients, making this an even more complex process. Thus, researchers are constantly searching for new strategies that are stable, safe, and easily accessible for stem cells, maintaining their potential to differentiate into several lineages.

Encapsulating cells in biodegradable micron-scale hydrogels offers numerous attractive features for tissue engineering, such as a highly hydrated tissue-like environment for cell and tissue repair, and the ability to form in vivo. The efficacy of cell-based therapies depends on materials science. Many materials properties have to be considered for the design of a hydrogel-based scaffold that mimics the extracellular matric, such as mechanical properties, degradation, and mass transfer and diffusion. Moreover, the fabrication process is critical for maintaining cell function and also is closely linked to the crosslinked structure of the hydrogel. All these considerations have posed a deep barrier for selectively choosing suitable materials for cell encapsulation. The main biopolymers that can be used to form a gel that mimics the extra-cellular matrix are alginate, gelatin, collagen, and fibrin. Most of these biopolymers need to be functionalized before forming a delivery vehicle for cells, which could potentially largely damaged cell viability. Furthermore, large quantities are essential for clinical application, where the amount required gram of cell-laden microgels that are not easy to fabricate under the current strategy. All these hindrances need to be removed before the broad application of cell-laden microgel for cell therapy.

In this Research Topic, we present a state-of-the-art study for the investigation of the therapeutic effect of various stem-cell-based therapy for various diseases treatment. There are totally three main subjects on cell-based therapy. First, we have materials, which are mainly hydrogels or their derived materials for encapsulating, delivering, and regulating the cell and 
cell behavior. For example, Sivaraj et al. have summarized the current hydrogel-based materials. Yang et al. have introduced an injectable glycol chitosan/dibenzaldehyde terminated PEG for cartilage defect repair. Lewicka et al. has employed silk protein matrices to regulate the differentiation of neural stem cells. Xu et al. have shown the hDPSCs for pancreatic disease, Second, there is the new encapsulation approach. Shao et al. have introduced a microfluidic-based technique for cell encapsulation. Third, there is the new delivery system; Li and Liu have engineered bacterial outer membrane vesicles as the new delivery platform. We also present the importance of considering designing biodegradable biomaterials for cell encapsulation and highlight recent advances in hydrogel design and their application. We are confident that the studies collected in this issue establish a new benchmark for the solution and new technologies for reprogramming stem cells as well as immune cells for a deeper understanding of stem cell differentiation, stem-cell-based tissue regeneration, and their stem cell recovery mechanisms.

\section{AUTHOR CONTRIBUTIONS}

All authors listed have made a substantial, direct, and intellectual contribution to the work and approved it for publication.

Conflict of Interest: The authors declare that the research was conducted in the absence of any commercial or financial relationships that could be construed as a potential conflict of interest.

Publisher's Note: All claims expressed in this article are solely those of the authors and do not necessarily represent those of their affiliated organizations, or those of the publisher, the editors and the reviewers. Any product that may be evaluated in this article, or claim that may be made by its manufacturer, is not guaranteed or endorsed by the publisher.

Copyright (c) 2021 Zhang and Wang. This is an open-access article distributed under the terms of the Creative Commons Attribution License (CC BY). The use, distribution or reproduction in other forums is permitted, provided the original author(s) and the copyright owner(s) are credited and that the original publication in this journal is cited, in accordance with accepted academic practice. No use, distribution or reproduction is permitted which does not comply with these terms. 